\title{
A Cellular Mechanism for the Transformation of a Sensory Input into a Motor Command
}

\author{
Gonzalo Viana Di Prisco, ${ }^{2}$ Edouard Pearlstein, ${ }^{2}$ Didier Le Ray, ${ }^{2}$ Richard Robitaille, ${ }^{2}$ and Réjean Dubuc ${ }^{1,2}$ \\ ${ }^{1}$ Département de Kinanthropologie, Université du Québec à Montréal, Montréal, Québec, Canada H3C 3P8, and ${ }^{2}$ Centre \\ de Recherche en Sciences Neurologiques, Université de Montréal, Montréal, Québec, Canada H3C 3J7
}

\begin{abstract}
The initiation and control of locomotion largely depend on processing of sensory inputs. The cellular bases of locomotion have been extensively studied in lampreys where reticulospinal (RS) neurons constitute the main descending system activating and controlling the spinal locomotor networks. $\mathrm{Ca}^{2+}$ imaging and intracellular recordings were used to study the pattern of activation of RS neurons in response to cutaneous stimulation. Pressure applied to the skin evoked a linear input/output relationship in RS neurons until a threshold level, at which a depolarizing plateau was induced, the occurrence of which was associated with the onset of swimming activity in a semi-intact preparation. The occurrence of a depolarizing plateau was abolished by blocking the NMDA receptors that are located on RS cells.
\end{abstract}

Moreover, the depolarizing plateaus were accompanied by a rise in $\left[\mathrm{Ca}^{2+}\right]_{\mathrm{i}}$, and an intracellular injection of the $\mathrm{Ca}^{2+}$ chelator BAPTA into single RS cells abolished the plateaus, suggesting that the latter are $\mathrm{Ca}^{2+}$ dependent and rely on intrinsic properties of RS cells. The plateaus were shown to result from the activation of a $\mathrm{Ca}^{2+}$-activated nonselective cation current that maintains the cell in a depolarized state. It is concluded that this intrinsic property of the RS neuron is then responsible for the transformation of an incoming sensory signal into a motor command that is then forwarded to the spinal locomotor networks.

Key words: brainstem; reticulospinal neurons; skin stimulation; sensorimotor integration; trigeminal nerve; $\mathrm{Ca}^{2+}$ imaging; lamprey
Animals must adapt their locomotion to the goal they seek and to the obstacles they encounter in their environment. For this reason, processing of sensory inputs is vital for the initiation and control of ongoing locomotion. In human, cat, and fish, cutaneous inputs mediate adaptive responses during locomotion in natural environments (Duysens, 1977; for review, see Grillner, 1985; Drew et al., 1996). Moreover, locomotor behaviors can be initiated and stopped by inputs of sensory origin (Viala et al., 1978; Clarke and Roberts, 1984; Boothby and Roberts, 1992) that interact with the motor command at various levels of the CNS (for review, see Rossignol, 1996). Although largely studied at the local network level in many vertebrate and invertebrate models (Büschges and El Manira, 1998), little is known about the cellular mechanisms of sensorimotor integration in the brainstem and higher structures.

In lamprey, reticulospinal (RS) axons are known to make direct connections onto spinal motoneurons (Buchanan and Cohen, 1982) and interneurons that constitute the fundamental neural circuitry for the generation of the basic locomotor pattern (Grillner et al., 1991). Anatomical and electrophysiological studies showed that dorsal column afferent fibers, carrying somesthetic information from the body, ascend to the brainstem and project disynaptically via a brainstem relay to RS neurons (Dubuc et al., 1993a,b). Similarly, cutaneous inputs from the head region are conveyed by the trigeminal system to RS neurons via a disynaptic pathway (Viana Di Prisco et al., 1995). Touching a lamprey over the head or

Received May 2, 2000; revised Aug. 14, 2000; accepted Aug. 14, 2000.

This work was supported by a Group Grant (Neurological Sciences) from the Canadian Medical Research Council, as well as from Fonds pour la Formation des Chercheurs et l'Aide à la Recherche (Québec). G.V.D.P. received a visiting professorship from the Fonds de la Recherche en Santé du Québec (FRSQ) (Québec). E.P received a fellowship from the Ministère de l'Éducation du Québec (MEQ) and the Jasper/Cordeau program. D.L.R. was a fellow from Institut National de la Santé et de la Recherche Médicale, the MEQ, and the Fondation de l'Université du Québec à Montréal. R.R. was a Junior II Scholarship of FRSQ. We thank D. Veilleux and S. Lepage for their technical assistance. We are grateful to Dr. L.-N. Hazrati for her participation in some of the imaging experiments and to Drs. L. Vinay and G. Scott for their comments on this manuscript. We also thank J. E. Gersmehl (U.S. Fish and Wildlife Service, Essex Junction, VT) for his kind supply of the lampreys.

Correspondence should be addressed to Dr. Réjean Dubuc, Université du Québec à Montréal, Département de Kinanthropologie, C.P. 8888, Succ. Centre-ville, Montréal, Québec, Canada H3C 3P8. E-mail: dubucr@physio.umontreal.ca.

Copyright (C) 2000 Society for Neuroscience $0270-6474 / 00 / 208169-08 \$ 15.00 / 0$ the body elicits a swimming response that allows the animal to move away from the stimulus (McClellan, 1988; Cardin et al., 1999). Because they integrate sensory information of various modalities and connect the spinal locomotor networks, RS neurons are likely to be of key importance for such an escape behavior. In a preliminary study, we showed that in some cases, tactile stimulation may elicit in lamprey RS neurons both a long-lasting NMDAdependent depolarization and a $\mathrm{Ca}^{2+}$ signal, which are accompanied by an escape swimming activity in a semi-intact preparation (Viana Di Prisco et al., 1997). These results indicate that RS neurons behave as command neurons that integrate sensory signals to trigger, when pertinent, an organized locomotor activity.

In the present study, calcium imaging and intracellular electrophysiological recordings were used to investigate in further details the pattern of activation and the integrative properties of individual RS cells. We describe the synaptic responses elicited in RS cells by cutaneous inputs from different body regions as well as a cellular mechanism responsible for the transformation of sensory inputs into a motor command.

\section{MATERIALS AND METHODS}

Electrophysiology. Experiments were performed in larval $(n=74)$ and young adult $(n=4)$ lampreys (Petromyzon marinus). All procedures conformed to the Canadian Medical Research Council guidelines and were approved by the University Animal Care and Use Committee. Under tricaine methanesulfonate (MS 222, $100 \mathrm{mg} / \mathrm{l}$ ) anesthesia, the animals were opened along the ventral midline and eviscerated. The dissection and experiments were performed in cold Ringer's with the following composition (in mM): $130 \mathrm{NaCl}, 2.1 \mathrm{KCl}, 2.6 \mathrm{CaCl}_{2}, 1.8 \mathrm{MgCl}_{2}, 4 \mathrm{HEPES}, 4$ dextrose, $1 \mathrm{NaHCO}_{3}$. The rostral end of the body up to the last gill was dissected isolating the brain and spinal cord, with the underlying cranium and notochord kept for support. The remaining body was left largely intact. In two experiments, a medial incision was made to expose the dorsal aspect of the spinal cord to the circulating Ringer's, but no differences were found with animals in which this incision was not made. The rostral end of the animal was pinned down to the Sylgard bottom of the experimental chamber perfused with oxygenated cold $\left(9^{\circ} \mathrm{C}\right)$ Ringer's, $\mathrm{pH} 7.4$, and a cut was made above the mesencephalon for decerebration purposes. The skin covering the remaining body was extended and pinned flat for better mechanical stimulation (see Fig. $1 A$ ). In 67 experiments, the skin covering the dorsal head region was left attached and also pinned flat to the Sylgard. In 12 preparations, the caudal two-thirds of the tail were left intact to freely swim behind, and insulated bipolar EMG electrodes (stainless steel wire diameter $50 \mu \mathrm{m}$; California Fine Wire Company, Grover Beach, CA) were 

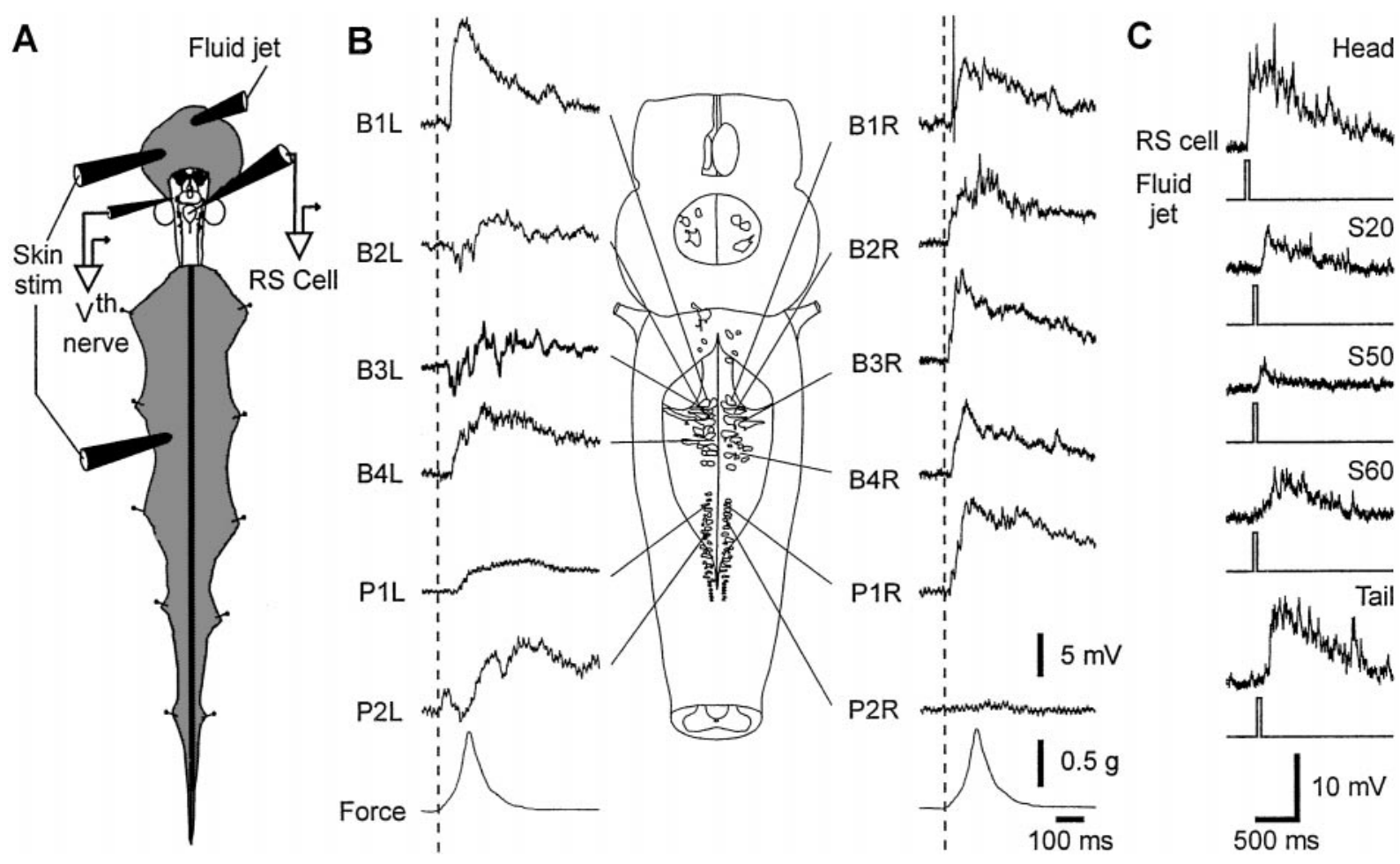

Figure 1. Synaptic responses of RS neurons to mechanical skin stimulation. A, Drawing of the experimental setup. Responses to skin stimulation or fluid jets were recorded intracellularly from RS neurons. Suction electrode recorded the afferent volley from the trigeminal nerve root (Vth nerve) in response to cutaneous head stimulation. $B$, Intracellular responses to the mechanical stimulation of the same skin spot in the head region with the same stimulation force were recorded successively in 12 identified RS neurons in both the MRRN [the nomenclature B1-B4 is the same as used by Rovainen (1982)] and the PRRN (P1 and P2). The bottom traces display the force applied to the skin. $C$, Responses evoked in an RS neuron to fluid jets of constant amplitude applied to the skin of head, at segments 20,50, and 60 (ipsilateral side) and at the tail.

inserted into the myotomes with an inter-electrode distance of $3 \mathrm{~mm}$. Two pairs of EMG electrodes were usually placed one on each side of the body at segmental levels $20-25$ of 100 .

RS neurons in the anterior (ARRN, $n=4)$, middle (MRRN, $n=134$, including two visually located Mauthner cells), and posterior (PRRN, $n=$ 44) rhombencephalic reticular nuclei, and in the mesencephalic reticular nucleus (MRN, $n=6$ ), were impaled under visual inspection with sharp glass micropipettes $(4 \mathrm{M}$ K-acetate, $>100 \mathrm{M} \Omega$ ). Mechanical stimulation to the skin was delivered (1) by a glass rod probe (diameter $0.5 \mathrm{~mm}$, kept submerged in the bath long enough to prevent thermal differences between the probe and the bathing solution) attached to a strain gauge either manually or by a computer-controlled step motor, and/or (2) by a Ringer's jet delivered through a micropipette (loaded with Ringer's taken from the bath) by positive pressure applied using a Picospritzer (see Fig. 1A). Suction electrodes monitored electrical activity of trigeminal nerves or ventral roots within the spinal cord. To assess whether the stimulation was activating cutaneous receptors, a series of tests were performed in 12 animals. (1) An area of $1 \mathrm{~cm}^{2}$ of skin was removed by performing a superficial incision and carefully dissecting the skin from the tissues below the dermis. (2) The dorsal columns were bilaterally cut at segment 10, and the extent of the lesion was later verified on histological transverse sections of the spinal cord. (3) Xylocaine, mixed with fast green for visualization, was locally applied to a patch of skin to inactivate cutaneous receptors. Drugs such as xylocaine, 6-cyano-7-nitroquinoxaline-2,3-dione (CNQX), 2-amino-5-phosphonopentanoate (AP-5), dizocilpine (MK-801), and flufenamic acid (FFA) were dissolved in fresh Ringer's, and either bathapplied or applied locally by positive pressure pulses of different duration $(10-100 \mathrm{msec})$ from a patch micropipette (the concentrations used are indicated in the text). In some experiments, BAPTA was intracellularly injected into the recorded RS neurons. All drugs were purchased from Sigma-Aldrich (Oakville, Ontario). After each drug application, a washout period of varying duration from several minutes to $>1 \mathrm{hr}$, depending on the specific drug, was allowed for recovery.

$\mathrm{Ca}^{2+}$ imaging. The preparation consisted of the isolated brain and rostral segments of the spinal cord $(n=20)$. The spinal cord was sectioned at segmental level 1 or 2 , and $\mathrm{Ca}^{2+}$ Green-Dextran $(10,000 \mathrm{MW}$, Molecular Probes, Eugene, Oregon) crystals were placed on the rostral cut end to permit in vitro retrograde transport of the dye in the dark, usually for $48 \mathrm{hr}$ (range $24-72 \mathrm{hr}$ ), while the preparation was perfused with Ringer's. The preparation was then transferred and pinned down at the bottom of a small chamber perfused with oxygenated cold Ringer's throughout the experiment, which normally lasted 3-6 hr. Labeled RS cells were imaged on a Nikon epifluorescent microscope and recorded with an intensified CCD camera (Hamamatsu C2400, Bridgewater, NJ; neutral density filter at
$50 \%$ ) at a rate of one to two images per second using IMAGE 1 computer system. Some recordings $(n=9)$ were made with a confocal microscope (Bio-Rad 600, Mississauga, Ontario). The $488 \mathrm{~nm}$ line of an Ion-Argon laser was attenuated to $1 \%$ of maximal power with a neutral density filter, and emitted fluorescence was detected through a long-pass filter at $515 \mathrm{~nm}$. Recording sites (somata, axons, and proximal dendrites) were identified and delineated for measurement on the monitor under mouse control. $\mathrm{Ca}^{2+}$ responses were expressed as relative changes in fluorescence $\Delta F / F$ $(\%)$, whereas the changes in $\mathrm{Ca}^{2+}$ dynamics were assessed by the time course of the signals. To quantify and compare $\mathrm{Ca}^{2+}$ responses between cells, the time series were imported to a spreadsheet, and the corresponding peaks, expressed as $\Delta F / F(\%)$, and areas, expressed in arbitrary units, were calculated. These values were used for statistical testing. In those experiments that required mechanical stability of the preparation, afferent signals were produced by electrical stimulation of the trigeminal nerve using single shocks delivered through a monopolar tungsten electrode (4-5 $\mathrm{M} \Omega$; Micro Probe, Clarksburg, MD).

\section{RESULTS}

\section{Synaptic responses of RS neurons to skin stimulation}

RS neurons trigger motor behavior in response to tactile stimulation in lampreys (Viana Di Prisco et al., 1997). Questions arose from this finding such as whether all RS neurons responded to tactile stimulation applied at different points along the body and the head and relative to the cellular mechanisms involved in triggering the motor behavior.

Most of the responses were depolarizing, but sometimes mixed depolarizing and hyperpolarizing postsynaptic potentials were observed (Fig. $1 B$ ). The shape and amplitude of the responses varied from one RS cell to another, even when located in the same reticular nucleus (Fig. $1 B$, compare the responses of $P 1 L$ and $P 2 L$ ). All RS neurons tested in each of the four reticular nuclei responded to skin stimulation (not shown for ARRN and MRN neurons), whether the stimulus was mechanical pressure or a fluid jet directed to the skin surface. In some experiments in which the pressure exerted by the glass rod on the skin was applied manually, responses to both ipsilateral and contralateral stimulation were studied, and those from the contralateral side were slightly larger in 

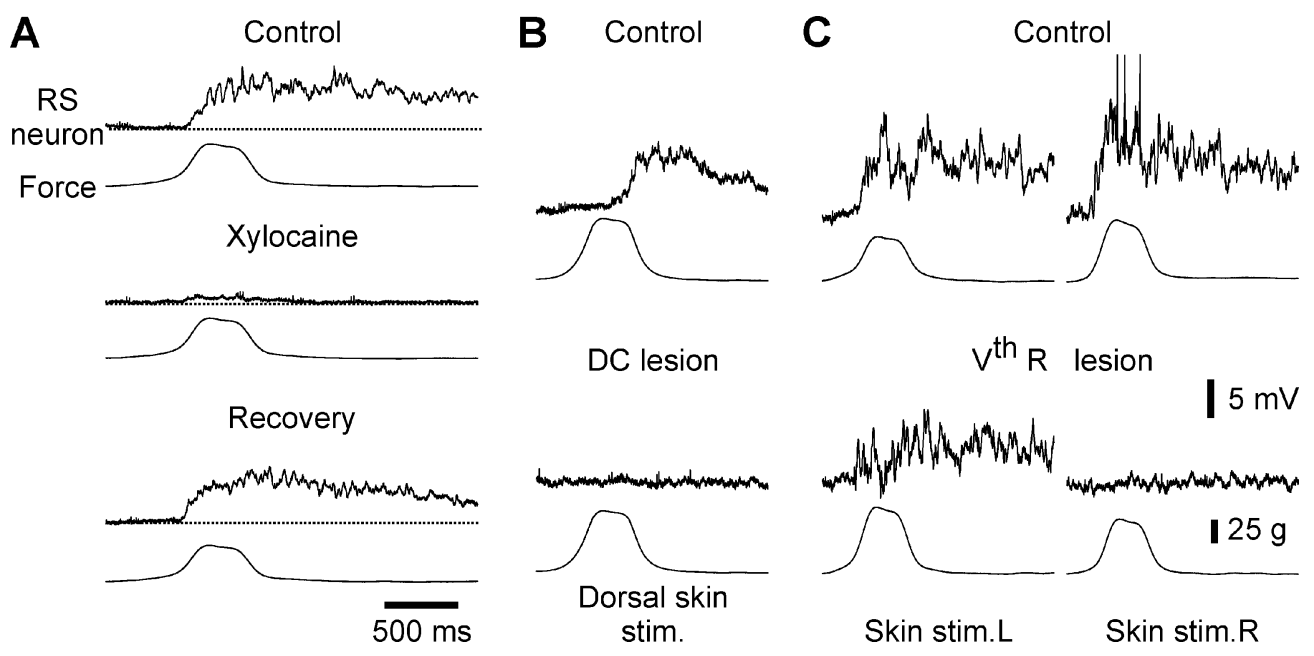

Figure 2. Cutaneous inputs are conveyed by dorsal columns or trigeminal nerves. $A$, Local application of xylocaine $(1 \%)$ onto the skin reversibly abolishes the synaptic responses of an $\mathrm{RS}$ neuron to mechanical pressure applied to that skin region (Force). B, Effects of a selective transection of the dorsal columns (bottom traces) on the synaptic responses evoked in an RS cell by skin stimulation applied at midbody. $C$, A section of the right trigeminal nerve (bottom traces) abolishes the responses evoked in an RS neuron by mechanical stimulation of the skin on the right side of the head (right) but not that on the left side (left). Same calibration in $A-C$.
MRRN neurons (data not shown). For the same stimulation intensity, the size of the responses varied in relation to the part of the body that was stimulated (Fig. $1 C$ ). Responses evoked by stimulation of either the head or the tail were comparable in amplitude, and three or four times larger than those elicited by mid-body stimulation ( $n=4$ cells; $p<0.01$; ANOVA). The same pattern of response was observed with mechanical application of pressure to the skin (data not illustrated). These results indicate that the reticulospinal system seems capable of discriminating spatially the sensory information arising from the different regions of the body.

A series of control experiments was performed to confirm the cutaneous origin of the inputs. Xylocaine $(1 \%)$ applied by a micropipette ejection over the stimulated skin area abolished the responses of RS cells (Fig. $2 A$ ), as did the surgical removal of a skin patch over the stimulated area (data not shown). Furthermore, the synaptic responses elicited in RS cells by skin stimulation at midbody were abolished by a selective transection of the dorsal columns in the rostral spinal cord $(n=7)$ (Fig. $2 B, D C$ lesion). The responses elicited by mechanical stimulation on one side of the head were abolished by a lesion of the trigeminal nerve on that side, whereas responses evoked by the other side were not affected $(n=2)$ (Fig. 2C).

Mechanical pressure of increasing strength and duration applied to the same skin area elicited responses of increasing amplitude in a given RS neuron (Fig. $3 A$ ). At low intensities, the shape of the compound EPSP matched the temporal course of the force trace remarkably well, but with a delay (several tens of milliseconds) because the pathway involved is disynaptic (Viana Di Prisco et al., 1995). The afferent volley recorded from the trigeminal nerve occurred during the rising phase of the force trace and continued when the stimulus was maintained. As the stimulation intensity was increased further, a completely different response occurred in MRRN neurons. The latter consisted of a long-duration depolarizing plateau that considerably outlasted the stimulus duration (Fig. $3 B$ ), and furthermore, spikes were often superimposed on the large depolarizing plateaus. Consequently, the stimulus-response relationship (Fig. $3 C$ ), which was linear at low stimulation strength, abruptly jumped to a different range as the stimulus strength was increased further [see also Viana Di Prisco et al. (1997)]. Similar results were obtained when cutaneous stimulation was applied to the tail (data not shown).

The responses of MRRN cells to skin stimulation were also studied in a semi-intact preparation ( $n=12$ animals), where the caudal two-thirds of the body was left intact to visualize and record active swimming. In these experiments, repeated fluid jets elicited short-lasting depolarizations of the RS neurons that eventually developed into a plateau potential (Fig. 3D). The onset of the plateau and the cell firing were accompanied by active swimming that consisted of symmetrical undulations of the body on both sides. The EMG activity that was recorded on both sides at mid- body (segment 50) of the animal showed left-right alternation (Fig. $3 D$ ). The long-lasting depolarizing plateau and the swimming activity considerably outlasted the afferent discharges induced by the cutaneous stimulation (three consecutive bursts produced by the three fluid jets in Fig. $3 D$ ), suggesting that intrinsic cellular properties, rather than a sensory-supported mechanism, were involved. We investigated further the mechanisms underlying the sustained depolarization in MRRN cells.

\section{Effects of NMDA receptor antagonists}

The presence of the abrupt break in the linearity of the stimulusresponse curve suggested a possible involvement of NMDA receptors, the activation of which is known to elicit nonlinearity in the synaptic responses (for review, see Daw et al., 1993). NMDA receptor antagonists were used to test this hypothesis. In two experiments, the noncompetitive NMDA receptor antagonist MK801 was applied locally onto the recorded RS cells. Figure 4 shows one example where the intensity of the mechanical stimulation on the surface of the snout was set to initiate a large depolarizing plateau in the recorded $\mathrm{RS}$ neuron (Fig. 4A, Control). After pressure-ejecting MK-801 (1 mM in pipette), a stimulus at the same intensity could not induce a depolarizing plateau. Only a small amplitude non-NMDA-mediated depolarization occurred, which did not outlast the duration of the stimulus. The response increased steadily with the stimulus strength, but no depolarizing plateau was ever observed after application of the drugs. This is exemplified by the stimulus-response relationship that remained linear under MK-801 (bath-applied $50 \mu \mathrm{M} ; n=3$ ) (Fig. 4B, ○), even at intensities largely exceeding those needed to induce a plateau under control conditions $(\mathbf{0})$. Similar results were obtained with local application of AP-5 (1 mM in pipette; $n=5$; data not shown) and bath application of AP-5 (50-200 $\mu \mathrm{M}, n=10$; data not shown) [see also Viana Di Prisco et al. (1997)].

\section{Role of the $\mathrm{Ca}^{2+}$ signal evoked by cutaneous stimulation}

NMDA receptors are largely permeable to $\mathrm{Ca}^{2+}$ (Ascher and Nowak, 1988), and a possible intracellular messenger role for $\mathrm{Ca}^{2+}$ in the depolarizing plateaus was studied using $\mathrm{Ca}^{2+}$ imaging techniques. In response to mechanical pressure or fluid jets directed to the surface of the snout, RS neurons loaded with Calcium Green Dextran $(n=20)$ displayed a large and sustained increase in relative fluorescence [see also Viana Di Prisco et al. (1997)]. This increase was up to $80 \%$ and slowly returned to control values $(>100$ sec). Intracellular recordings, performed simultaneously with $\mathrm{Ca}^{2+}$ imaging $(n=3)$, demonstrated that the increase in intracellular $\mathrm{Ca}^{2+}$ was always associated with the development of a depolarizing plateau in the recorded RS neuron (Fig. $5 A$ ). In control conditions, light electrical stimulation of one trigeminal nerve only 
A

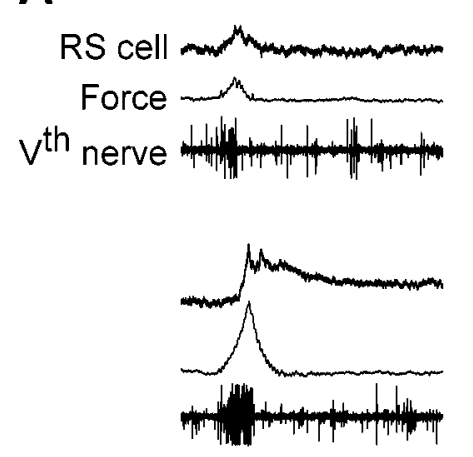

C

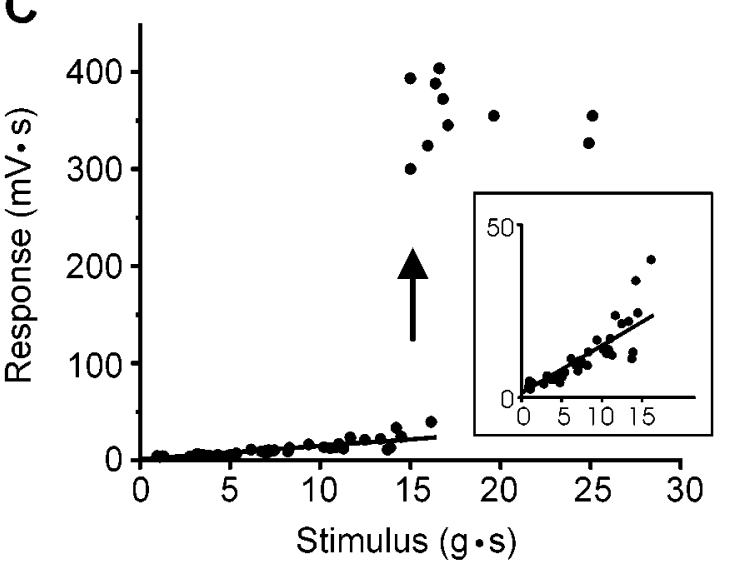

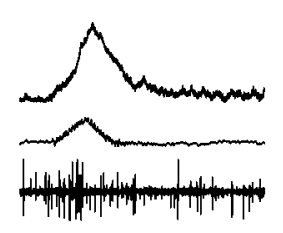
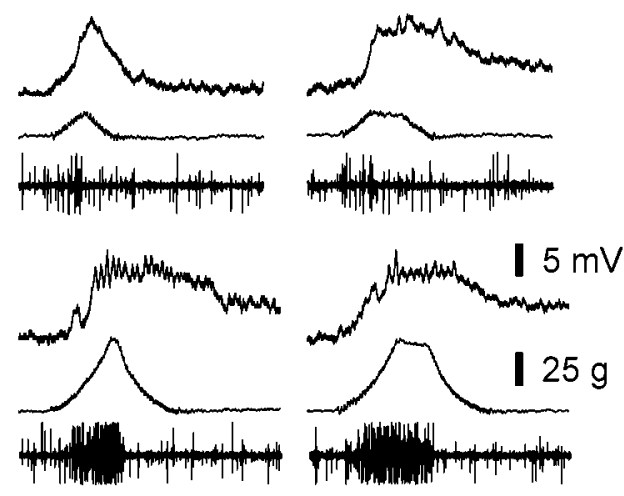

$1 \mathrm{~s}$
B
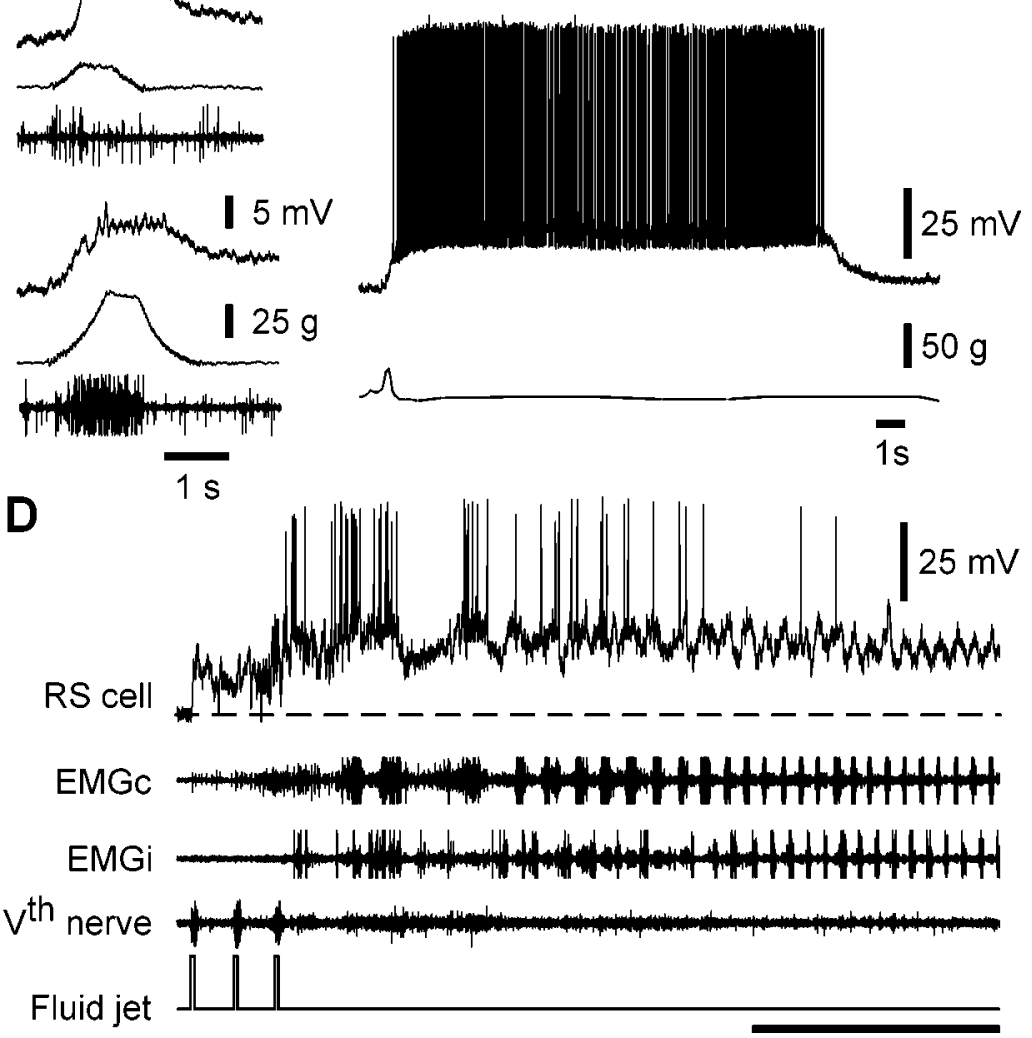

$5 \mathrm{~s}$

Figure 3. Responses to mechanical stimulation of the head. A, Responses evoked in an RS neuron (top traces) by mechanical stimulation of different intensity and duration (Force, middle traces). The afferent discharge in the trigeminal nerve (Vth nerve) are also shown (bottom traces). B, Suprathreshold mechanical stimulation induces a large and long-lasting depolarizing plateau. $C$, Plot of the stimulus-response relationship in one RS neuron. Areas under the synaptic response $(\mathrm{mV} \cdot \mathrm{g})$ and the force of the stimulus $(\mathrm{g} \cdot \mathrm{s})$ were used. A linear relationship is observed for low intensities of stimulation (inset). $D$, Response to repeated low-intensity fluid jets, directed to the snout in a semi-intact preparation. The plateau potential was accompanied by bursts of activity alternating between ipsilateral and contralateral ( $E M G i$ and $E M G c$, respectively, recorded at segment 50 ) sides of the body. $A, B, C$, and $D$ from different cells.
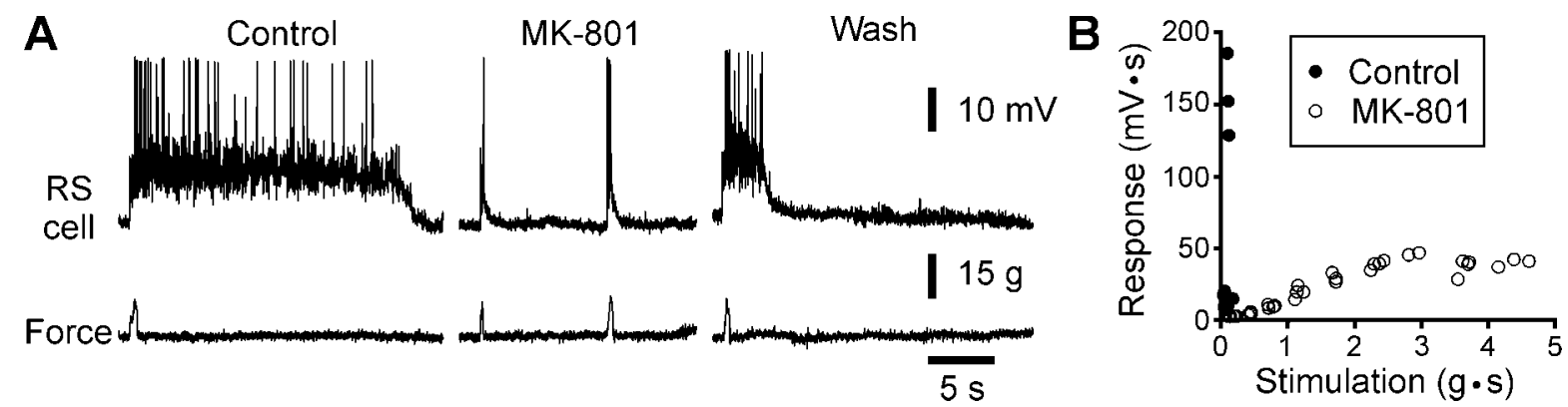

Figure 4. NMDA antagonists prevent the depolarizing plateaus from occurring. $A$, Local application of the noncompetitive NMDA receptor antagonist MK-801 (1 mM) prevents the occurrence of a plateau in response to mechanical stimulation of the snout (Force). B. Plot of the stimulus-response relationship in control $(\mathbf{O})$ and after bath perfusion of $50 \mu \mathrm{M}$ MK-801 $(\bigcirc)$. Areas under the synaptic response $(\mathrm{mV} \cdot \mathrm{g})$ and the force of the stimulus $(\mathrm{g} \cdot \mathrm{s})$ were used.

elicited a depolarization of small amplitude and short duration (first trace), with no significant increase in fluorescence (bottom trace, thick line). As the stimulation intensity was increased, a sustained depolarizing plateau occurred (second and third traces), accompanied by a large increase in fluorescence. The concurrence of both the depolarizing plateau and the $\mathrm{Ca}^{2+}$ signal suggested that plateau potentials might result from the increase in the intracellular $\mathrm{Ca}^{2+}$ concentration. In the presence of AP-5 $(n=3)$, both the depolarizing plateau (middle traces) and the increase in fluorescence (bottom trace, thin line) were abolished, indicating that the intracellular $\mathrm{Ca}^{2+}$ signals were also dependent on the activation of NMDA receptors.

To test whether the depolarizing plateaus were linked to the intracellular rise in $\mathrm{Ca}^{2+}$, the $\mathrm{Ca}^{2+}$ chelator BAPTA was injected intracellularly into the recorded RS cells $(n=7)$. The depolarizing plateau that was elicited by skin mechanical stimulation under control conditions (Fig. 5B, left trace) was completely abolished by an intracellular injection of BAPTA into the recorded cell (100 $\mathrm{mM}$; middle trace). Even with an increased intensity $(n=5)$, the mechanical stimulation could not elicit a depolarizing plateau (right trace), and the stimulus-response relationship (Fig. 5C, $\bigcirc$ ) remained linear for a range of intensities larger than the one used to elicit a plateau in control conditions $(\mathbf{)})$. A detailed analysis of the size of the responses for the low stimulation strength $(n=3)$ (Fig. $5 D$ ) demonstrated that the mean response areas in control (filled bars) and in BAPTA-loaded neurons (empty bars) were quite 


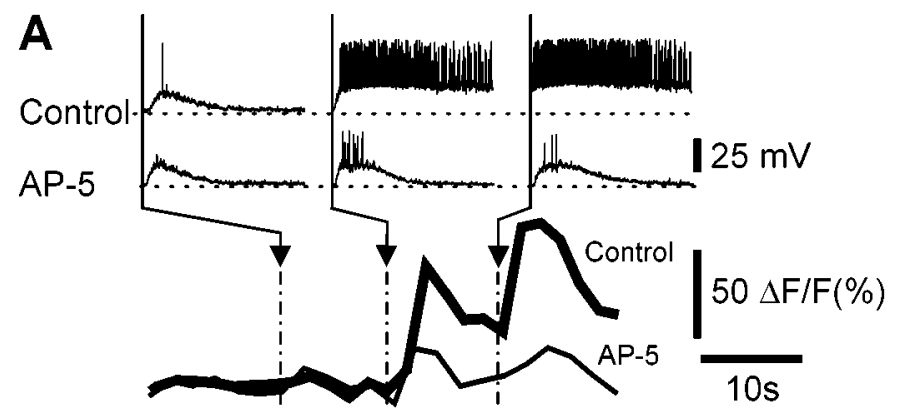

B
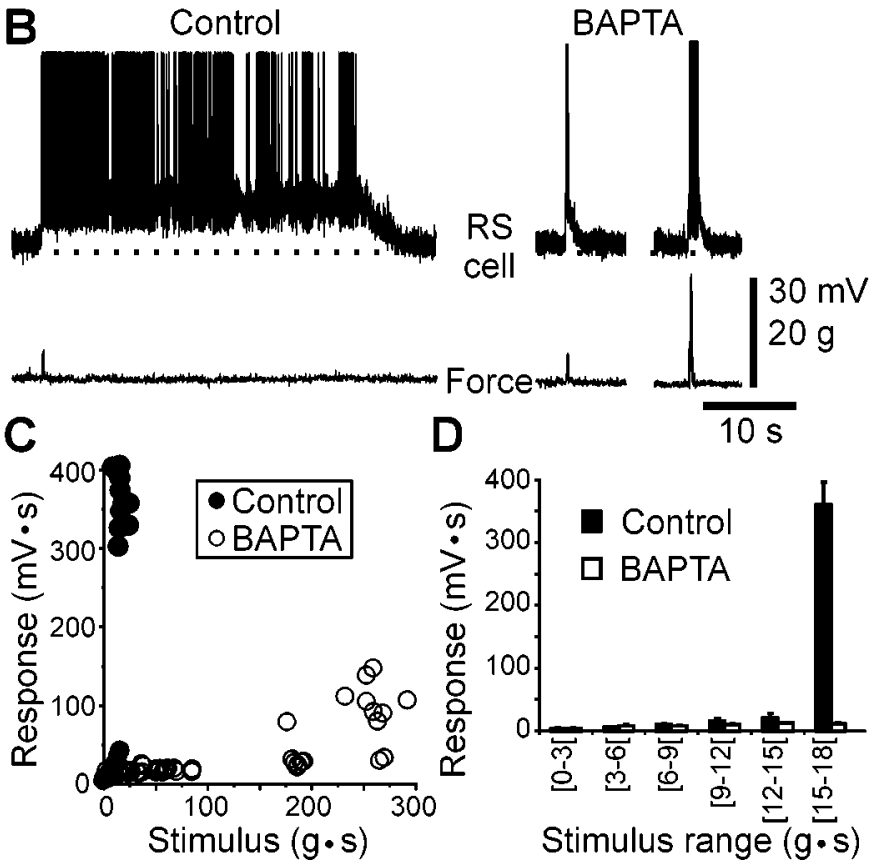

Figure 5. Role of $\left[\mathrm{Ca}^{2+}\right]_{\mathrm{i}}$ during plateau potentials. $A$, Simultaneous $\mathrm{Ca}^{2+}$ and intracellular recordings from an $\mathrm{RS}$ neuron during trigeminal electrical stimulation in control and after bath application of the competitive NMDA receptor antagonist AP-5 $(300 \mu \mathrm{M})$. Intracellular $\mathrm{Ca}^{2+}$ time course: thick line in Control, thin line under $A P$-5. Same time calibration for all traces. $B$, Intracellular responses evoked by skin mechanical stimulation (Force) in control and after intracellular injection of BAPTA (100 mM). The stimulus strength was increased fourfold on the right. $C$, Plot of the stimulus-response relationship from an RS cell in control (O) and when loaded with BAPTA $(O)$. $D$, Histogram illustrating the amplitude of the responses in control (filled column) and in BAPTA-loaded (empty column) RS neuron $(p<0.01, t$ test) for different ranges of stimulation strengths. Error bars illustrate the SEM.

similar. This suggested that the excitatory amino acid transmission was not modified by BAPTA. It was only at the stronger stimulus strengths $(>15 \mathrm{~g} \cdot \mathrm{s})$ that the responses were significantly decreased in BAPTA-loaded RS neurons (Fig. 5D) $(p<0.01 ; t$ test), indicating that the intracellular $\mathrm{Ca}^{2+}$ signal was only involved in the response to stronger stimuli (i.e., for the expression of the depolarizing plateau). The results after BAPTA injections in single RS cells indicate first, that the plateau properties are intrinsic to RS cells and second, that they rely on a $\mathrm{Ca}^{2+}$-dependent mechanism.

\section{Calcium-activated nonselective cation current-mediated plateau potentials}

Calcium-activated nonselective $(\mathrm{CAN})$ cation currents $\left(I_{\mathrm{CAN}}\right)$ have been shown to generate long-lasting depolarizing plateaus in several classes of neurons (Zhang et al., 1995; Fraser and MacVicar, 1996; Wilson et al., 1996; Congar et al., 1997; Klink and Alonso, 1997; Morisset and Nagy, 1999). To test whether the plateaus observed in RS cells resulted from the activation of such a current, the $I_{\text {CAN }}$ blocker FFA (Lee et al., 1996; Morisset and Nagy, 1999) was bath-applied $(n=3 ; 200 \mu \mathrm{M})$ or pressure-applied directly onto
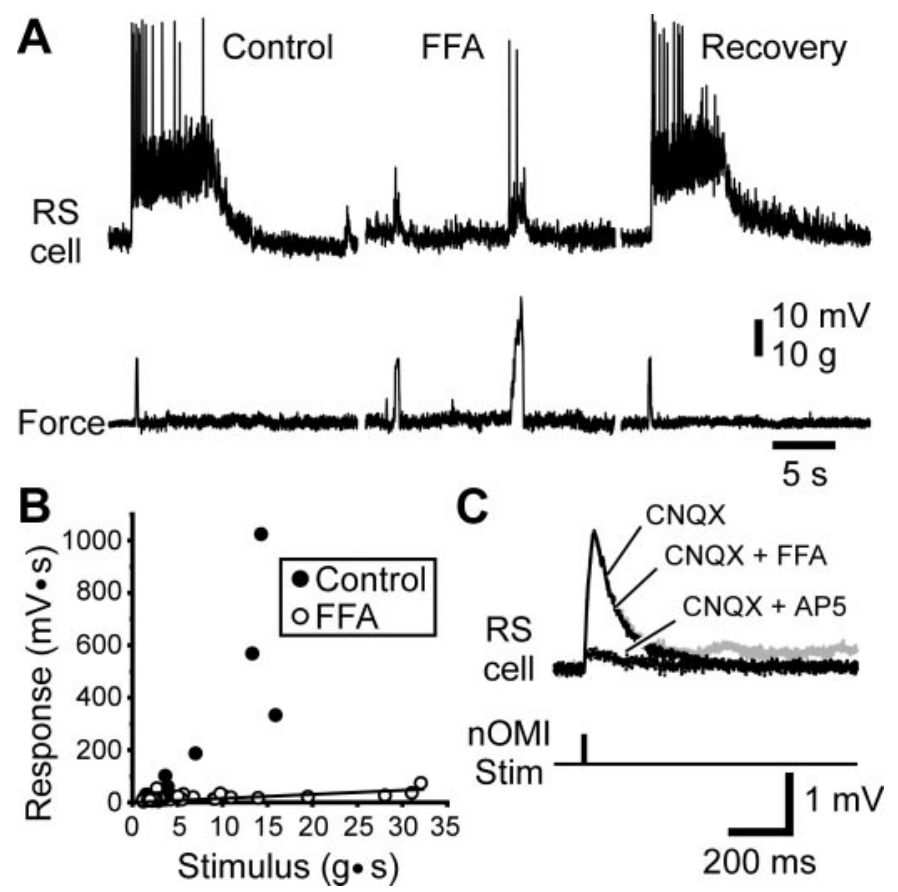

Figure 6. Effects of the $I_{\text {CAN }}$ blocker, flufenamic acid (FFA). A, Intracellular responses evoked in an RS neuron by skin stimulation (Force) in control and after local pressure ejection of FFA $(1 \mathrm{~mm})$. $B$, Plot of the stimulus-response relationship in control (O) and after FFA local application (O). $C$, Monosynaptic intracellular responses evoked in an RS neuron by electrical stimulation of axons originating from the intermediate octavomotorius nucleus (nOMI Stim) under bath application of CNQX (10 $\mu \mathrm{M}), \mathrm{CNQX}$ and FFA $(200 \mu \mathrm{M})$, or CNQX and AP-5 $(50 \mu \mathrm{M})$.

the recorded RS cells $(n=7 ; 1 \mathrm{~mm})$. In all cases, the drug abolished the depolarizing plateaus. Figure 6 illustrates one example where mechanical stimulation applied to the surface of the snout induced a depolarizing plateau in an RS neuron (Fig. 6 $\mathrm{A}$, Control). The local application of FFA abolished the plateau, and only a depolarization of small amplitude and short duration was then elicited ( $F F A$, first response). Increasing the stimulus strength evoked a larger depolarization with firing of action potentials, but no depolarizing plateau ( $F F A$, second response). Recovery was obtained several minutes after the application of FFA (Recovery). As for the previous experiments with the NMDA antagonists or BAPTA, the stimulus-response curve remained linear after local applications of FFA (Fig. 6B, $\bigcirc$ ), even for large stimulus intensities.

It was reported previously that FFA may have an effect on NMDA receptors (Lerma and Martin del Rio, 1992; Chen et al., 1998). To verify that the blockade of the depolarizing plateaus was not imputable to an alteration of the NMDA receptor-mediated response, electrical stimulation was applied to the axons issued from the ipsilateral intermediate octavomotor nucleus ( $\mathrm{OMMI})$, which make monosynaptic contacts with RS neurons, and the responses evoked in RS neurons were analyzed. These experiments were performed in the presence of CNQX $(10 \mu \mathrm{M})$ in $\mathrm{Mg}^{2+}$-free Ringer's $(n=5)$. The remaining monosynaptic EPSP was mediated by NMDA receptors because it was abolished by AP-5 $(50 \mu \mathrm{M})$ [see also Alford et al. (1995)]. Adding $200 \mu \mathrm{M}$ FFA to the perfusion saline had no effect on the NMDA-mediated EPSPs (Fig. 6C), as corroborated by the absence of significant change (ANOVA test, $p>0.05)$ in their peak amplitude and decay time constant estimated with a second order exponential decay fit (Table 1).

\section{DISCUSSION}

Results from the present study describe step by step the cellular mechanisms that are responsible for eliciting swimming in response to cutaneous inputs in lampreys. We showed that cutaneous inputs excite an ensemble of RS neurons that are known to make direct connections with the spinal cord locomotor networks and motoneu- 
Table 1. Effect of FFA on both the peak amplitude and the time constants of the NMDA component of the EPSP evoked by nOMI axon stimulation

\begin{tabular}{llll} 
& $\begin{array}{l}\text { Peak } \\
(\mathrm{msec})\end{array}$ & \multicolumn{2}{l}{ Time constants $(\mathrm{msec})$} \\
\cline { 3 - 4 } & $1.82 \pm 0.35$ & $212.5 \pm 93.1$ & $\tau 2$ \\
\hline Control & $1.84 \pm 0.44$ & $241.7 \pm 120.2$ & $207.7 \pm 98.2$ \\
FFA & $p>0.05$ & $p>0.05$ & $p>0.05$ \\
$p$ (ANOVA) & $p$
\end{tabular}

All values express the mean \pm the SEM.

rons. When the stimulus strength reaches a threshold level, the incoming sensory inputs activate NMDA receptors on RS cells, and a $\mathrm{Ca}^{2+}$-dependent depolarizing plateau results from the activation of an $I_{\mathrm{CAN}}$. Discharges that accompany the depolarizing plateaus in RS neurons activate the spinal locomotor networks, and swimming is generated.

\section{The transformation of a cutaneous input into a motor command}

It has been proposed that RS neurons of lampreys are descending command neurons that are responsible for the initiation of swimming (McClellan and Grillner, 1984; McClellan, 1987, 1988), and there is ample evidence to support such a role. RS cells receive inputs from different sensory afferents: vestibular (Bussières and Dubuc, 1992; Orlovsky et al., 1992), trigeminal (Viana Di Prisco et al., 1995), cutaneous from the body region (Dubuc et al., 1993a,b), olfactory (Rovainen, 1982), visual (Deliagina et al., 1993; Zompa and Dubuc, 1996), and lateral line systems (Deliagina et al., 1995). They also receive feedback information from the spinal locomotor networks that modulate their activity during locomotion (Dubuc and Grillner, 1989; Vinay and Grillner, 1992), and they relay inputs from a specific mesencephalic region that is homologous to the mesencephalic locomotor region described in other vertebrate species (Sirota et al., 1995). Their ubiquitous sources of inputs confer to lamprey RS cells a strategic location for the initiation and control of movement. An intermediate location between sensory neurons and central pattern generators has been discussed previously as an important feature for command neurons in invertebrates (Frost and Katz, 1996). We have not confirmed yet whether all RS cells behave as command neurons (the study of plateau properties being performed only on MRRN neurons). Nevertheless, we found that stimulation of a given skin area elicits synaptic responses in RS neurons of all four reticular nuclei, the largest responses occurring in the MRRN. This indicates that there is a divergence of specific cutaneous inputs to a large number of RS cells. Spatial discrimination on the other hand seems rather poor because responses in a single RS cell can be elicited by stimulation of different areas over the skin surface. Moreover, the fact that receptive fields of primary afferents can be fairly small (Matthews and Wickelgren, 1978; Christenson et al., 1988) suggests that significant convergence occurs at the RS level. As shown in other species (Le Ray et al., 1997), the divergence and convergence of sensory inputs onto a given neuron involved in motor control may be of great importance in the shaping of the output motor program. Similarly in lampreys, such a divergence/convergence organization may play a key role in shaping the escape response of the animal.

RS neurons displayed responses with varying amplitudes and patterns, depending on the region of the skin that was stimulated, and larger responses were obtained from the head and tail regions. The increased sensitivity of the head region may be important to correct the animal trajectory when hitting a forward obstacle, and the high sensitivity of the tail to allow the animal to respond faster by an escape reaction to possible attacks from behind. There was a remarkable linear relationship between the low-strength stimuli and the synaptic responses, suggesting that the RS neuron membrane potential behaves like a linear transducer of the forces applied to the skin. RS cells are thus closely linked with the

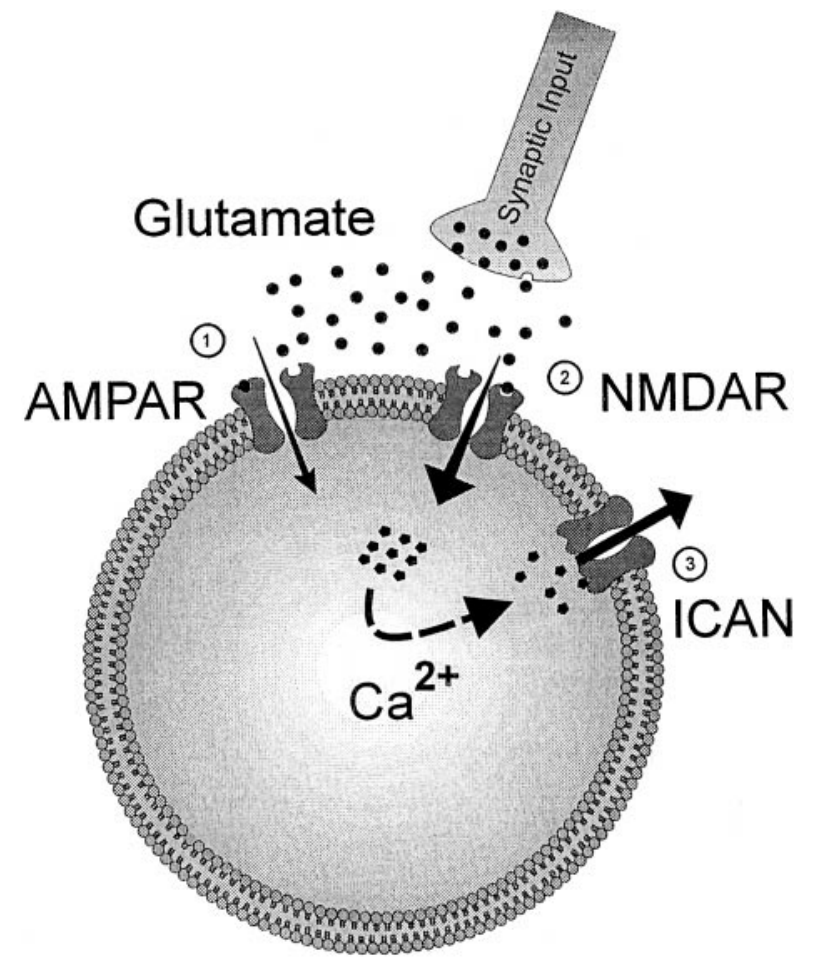

Figure 7. Schematic drawing of the cellular mechanisms that appear to be involved in the transformation of a sensory input into a motor command in lamprey reticulospinal neurons. Glutamate is released at the input synapse from the sensory relay onto the RS cell and activates both AMPA (1) and NMDA receptors (2). The simplest hypothesis would be that the $\mathrm{Ca}^{2+}$ entering the RS neuron through the NMDA receptor-channel activates an $I_{\text {CAN }}(3)$ that generates a depolarizing plateau.

periphery, and little transformation of the sensory input occurs at low stimulus intensity. Interestingly, however, the linearity breaks down with stronger stimuli, and the elicited response considerably outlasts the stimulus duration in MRRN neurons. Because depolarizing plateaus are accompanied by spiking activity that will raise the level of excitation of the spinal locomotor networks, this will lead to swimming in a semi-intact preparation. It constitutes a switch from a passive sensory response to an active motor-related activity in RS neurons [see also Viana Di Prisco et al. (1997)].

\section{Cellular mechanisms of plateau potentials in RS neurons}

Our work unraveled the cellular cascade of events that leads to the generation of depolarizing plateau potentials in MRRN neurons. The simplest cascade we can propose according to our results is the following (Fig. 7): the ligand activation of NMDA receptors leads to $\mathrm{Ca}^{2+}$ entry into the RS cell, which in turn activates an $I_{\mathrm{CAN}}$.

Plateau potentials induced by NMDA receptor activation have been reported previously in motoneurons (MacLean et al., 1997; Rioult-Pedotti, 1997; Grillner et al., 1998) and spinal interneurons (Kiehn et al., 1996). In all cases, those plateaus were regenerative and voltage dependent (Kiehn, 1991) and, as such, supported rhythmic activity (Grillner et al., 1998). This is not the case in MRRN cells where the cutaneous stimulation elicited a single long-lasting but nonregenerative depolarizing plateau. These differences could be attributed to the function of the cells involved. In motoneurons, NMDA-activated plateau potentials shape and stabilize rhythmic activity (Schmidt et al., 1998). In contrast, lamprey RS cells are command neurons, and as such, they need to generate a tonic increase in the activity of the spinal central pattern generators for locomotion, which will trigger a locomotor bout of sufficient duration to allow the animal to swim away from the stimulus.

We confirmed here that in response to low-intensity cutaneous or trigeminal nerve stimulation, the activation of the NMDA receptors located on RS cells results in brief transient $\mathrm{Ca}^{2+}$ responses 
[see also Viana Di Prisco et al. (1997)]. However, trains of pulses or repeated skin stimulation induced a clear summation that triggered a sustained rise in $\left[\mathrm{Ca}^{2+}\right]_{i}$. In the same way, the mechanical stimulation of the skin has also been shown to elicit sustained $\mathrm{Ca}^{2+}$ responses in Aplysia semi-intact preparation (Tsau et al., 1994).

The chelation of $\mathrm{Ca}^{2+}$ prevented the switch from sensory to motor-related response to occur in MRRN neurons, indicating that the depolarizing plateaus are $\mathrm{Ca}^{2+}$ dependent. However, it is difficult to ascertain to what extent the increase in $\left[\mathrm{Ca}^{2+}\right]_{\mathrm{i}}$ observed in this study is attributable either to $\mathrm{Ca}^{2+}$ entry through ligand or voltage-dependent channels or to the release from internal $\mathrm{Ca}^{2+}$ stores. It has been proposed that $\mathrm{Ca}^{2+}$ that enters through high- and low-threshold voltage-activated $\mathrm{Ca}^{2+}$ conductances contributes to both the depolarization and the termination of pharmacologically induced regenerative plateaus, by acting on $\mathrm{Ca}^{2+}$-dependent $\mathrm{K}^{+}$conductances (Grillner et al., 1998; Kiehn and Eken, 1998). This involvement of voltage-gated channels explains the voltage sensitivity of the sustained depolarization of the "classical" voltage-triggered plateau potentials. However, although possibly involved, such an activation of voltage-gated $\mathrm{Ca}^{2+}$ conductances did not seem to play a large role in the initiation of plateaus in RS neurons. Indeed, the burst of action potentials evoked in the absence of NMDA receptor activation (e.g., Fig. $5 A$, where a strong mechanical stimulation under AP-5 evoked a $2 \mathrm{sec}$ duration burst of spikes) was unable to elicit a depolarizing plateau. Moreover, Rouse et al. (1998) recently showed that injection of a depolarizing current into RS neurons did not trigger a sustained plateau, supporting the idea that voltage-activated $\mathrm{Ca}^{2+}$ channels do not play a significant role. Whether voltage-activated $\mathrm{Ca}^{2+}$ channels play a role in maintaining the depolarizing plateau by insuring the sustained activation of the CAN conductance remains to be determined, as does the possible role of other $\mathrm{Ca}^{2+}$ sources.

Using the $I_{\text {CAN }}$ blocker FFA, we have shown that a CAN current is responsible for the sustained depolarizing plateau. FFA induces a release from $\mathrm{Ca}^{2+}$ stores followed by a direct block of the CAN channel (Lee et al., 1996). It was shown recently to block the CAN channel in hippocampal CA1 neurons (Partridge and Valenzuela, 2000). The $I_{\text {CAN }}$ is classically described as a voltage-independent current (Partridge et al., 1994) and is involved in sustained depolarization in both invertebrate (Zhang et al., 1995; Wilson et al., 1996) and vertebrate neurons (Fraser and MacVicar, 1996; Congar et al., 1997; Klink and Alonso, 1997; Morisset and Nagy, 1999; but see Perrier and Hounsgaard, 1999). In such studies, the $I_{\mathrm{CAN}}$ could be activated by $\mathrm{Ca}^{2+}$ that enters the cell through voltage-gated channels in response to either synaptic or direct electrical stimulation of the neurons. However, in most neuronal systems, the CAN current needed external modulatory influence to be expressed (Fraser and MacVicar, 1996; Congar et al., 1997). In contrast, in MRRN neurons, $I_{\mathrm{CAN}}$ represents the normal cellular mechanism involved in the integration of the incoming sensory information, and to our knowledge, it is the first demonstration of its involvement in command generation in the CNS.

In conclusion, lamprey RS cells display complex membrane potential dynamics that play a key role in the generation of motor commands. Such properties may be generally present in motor command neurons, allowing short-lasting sensory inputs to be transformed into a sustained activation of these neurons. They may also be of importance for sensorimotor transformations in mammalian brain.

\section{REFERENCES}

Alford S, Zompa I, Dubuc R (1995) Long-term potentiation of glutamatergic pathways in the lamprey brainstem. J Neurosci 15:7528-7538.

Ascher P, Nowak L (1988) The role of divalent cations in the $N$-methylD-aspartate responses of mouse central neurones in culture. J Physiol (Lond) 399:247-266.

Boothby KM, Roberts A (1992) The stopping response of Xenopus laevis embryos: pharmacology and intracellular physiology of rhythmic spinal neurones and hindbrain neurones. J Exp Biol 169:65-86.

Buchanan JT, Cohen AH (1982) Activities of identified interneurons, motoneurons, and muscle fibers during fictive swimming in the lamprey and effects of reticulospinal and dorsal cell stimulation. J Neurophysiol 47:948-960.

Büschges A, El Manira A (1998) Sensory pathways and their modulation in the control of locomotion. Curr Opin Neurobiol 8:733-739.

Bussières N, Dubuc R (1992) Phasic modulation of transmission from vestibular inputs to reticulospinal neurons during fictive locomotion in lampreys. Brain Res 582:147-153.

Cardin S, Le Ray D, Robitaille R, Dubuc R (1999) Motor responses elicited by skin stimulation in lampreys. Soc Neurosci Abstr 25:1906.

Chen Q, Olney JW, Lukasiewicz PD, Almli T, Romano C (1998) Fenamates protect neurons against ischemic and excitotoxic injury in chick embryo retina. Neurosci Lett 242:163-166.

Christenson J, Boman A, Lagerback PA, Grillner S (1988) The dorsal cell, one class of primary sensory neuron in the lamprey spinal cord. I. Touch, pressure but no nociception: a physiological study. Brain Res 440:1-8.

Clarke JD, Roberts A (1984) Interneurones in the Xenopus embryo spinal cord: sensory excitation and activity during swimming. J Physiol (Lond) 354:345-362.

Congar P, Leinekugel X, Ben-Ari Y, Crepel V (1997) A long-lasting $\mathrm{Ca} 2+$-activated nonselective cationic current is generated by synaptic stimulation or exogenous activation of group I metabotropic glutamate receptors in CA1 pyramidal neurons. J Neurosci 17:5366-5379.

Daw NW, Stein PS, Fox K (1993) The role of NMDA receptors in information processing. Annu Rev Neurosci 860:207-222.

Deliagina TG, Grillner S, Orlovsky GN, Ullén F (1993) Visual input affects the response to roll in reticulospinal neurons of the lamprey. Exp Brain Res 95:421-428.

Deliagina T, Ullén F, Gonzalez M, Ehrsson H, Orlovsky G, Grillner S (1995) Initiation of locomotion by lateral line photoreceptors in lamprey: behavioural and neurophysiological studies. J Exp Biol 198:2581-2591.

Drew T, Cabana T, Rossignol S (1996) Responses of medullary reticulospinal neurones to stimulation of cutaneous limb nerves during locomotion in intact cats. Exp Brain Res 111:153-168.

Dubuc R, Grillner S (1989) The role of spinal cord inputs in modulating the activity of reticulospinal neurons during fictive locomotion in the lamprey. Brain Res 483:196-200.

Dubuc R, Bongianni F, Ohta Y, Grillner S (1993a) Anatomical and physiological study of brainstem nuclei relaying dorsal column inputs in lampreys. J Comp Neurol 327:260-270.

Dubuc R, Bongianni F, Ohta Y, Grillner S (1993b) Dorsal root and dorsal column mediated synaptic inputs to reticulospinal neurons in lampreys: involvement of glutamatergic, glycinergic, and GABAergic transmission. J Comp Neurol 327:251-269.

Duysens J (1977) Reflex control of locomotion as revealed by stimulation of cutaneous afferents in spontaneously walking premammillary cats. J Neurophysiol 40:737-751.

Fraser DD, MacVicar BA (1996) Cholinergic-dependent plateau potential in hippocampal CA1 pyramidal neurons. J Neurosci 16:4113-4128.

Frost WN, Katz PS (1996) Single neuron control over a complex motor program. Proc Natl Acad Sci USA 93:422-426.

Grillner S (1985) Neurobiological bases of rhythmic motor acts in vertebrates. Science 228:143-149.

Grillner S, Ekeberg O, El Manira A, Lansner A, Parker D, Tegner J, Wallén P (1998) Intrinsic function of a neuronal network: a vertebrate central pattern generator. Brain Res Rev 26:184-197.

Grillner S, Wallén P, Brodin L, Lansner A (1991) Neuronal network generating locomotor behavior in lamprey: circuitry, transmitters, membrane properties, and simulation. Annu Rev Neurosci 14:169-199.

Kiehn O (1991) Plateau potentials and active integration in the "final common pathway" for motor behaviour. Trends Neurosci 14:68-73.

Kiehn O, Eken T (1998) Functional role of plateau potentials in vertebrate motor neurons. Curr Opin Neurobiol 8:746-752.

Kiehn O, Johnson BR, Raastad M (1996) Plateau properties in mammalian spinal interneurons during transmitter-induced locomotor activity. Neuroscience 75:263-273.

Klink R, Alonso A (1997) Ionic mechanisms of muscarinic depolarization in entorhinal cortex layer II neurons. J Neurophysiol 77:1829-1843.

Le Ray D, Clarac F, Cattaert D (1997) Functional analysis of the sensory motor pathway of resistance reflex in crayfish. II. Integration Of sensory inputs in motor neurons. J Neurophysiol 78:3144-3153.

Lee RJ, Shaw T, Sandquist M, Partridge LD (1996) Mechanism of action of the non-steroidal anti-inflammatory drug flufenamate on $\left[\mathrm{Ca}^{2+}\right]_{\mathrm{i}}$ and $\mathrm{Ca}(2+)$-activated currents in neurons. Cell Calcium 19:431-438.

Lerma J, Martin del Rio R (1992) Chloride transport blockers prevent $N$-methyl-D-aspartate receptor-channel complex activation. Mol Pharmacol 41:217-222.

MacLean JN, Schmidt BJ, Hochman S (1997) NMDA receptor activation triggers voltage oscillations, plateau potentials and bursting in neonatal rat lumbar motoneurons in vitro. Eur J Neurosci 9:2702-2711.

Matthews G, Wickelgren WO (1978) Trigeminal sensory neurons of the sea lamprey. J Comp Physiol 123:329-333.

McClellan AD (1987) In vitro CNS preparations: unique approaches to the study of command and pattern generation systems in motor control. J Neurosci Methods 21:251-264.

McClellan AD (1988) Brainstem command systems for locomotion in the lamprey: localization of descending pathways in the spinal cord. Brain Res 457:338-349. 
McClellan AD, Grillner S (1984) Activation of "fictive swimming" by electrical microstimulation of brainstem locomotor regions in an in vitro preparation of the lamprey central nervous system. Brain Res 300:357-361.

Morisset V, Nagy F (1999) Ionic basis for plateau potentials in deep dorsal horn neurons of the rat spinal cord. J Neurosci 19:7309-7316.

Orlovsky GN, Deliagina TG, Wallén P (1992) Vestibular control of swimming in lamprey. I. Responses of reticulospinal neurons to roll and pitch. Exp Brain Res 90:479-488.

Partridge LD, Valenzuela CF (2000) Block of hippocampal CAN channels by flufenamate. Brain Res 867:143-148.

Partridge LD, Muller TH, Swandulla D (1994) Ca2+-activated nonselective channels in the nervous system. Brain Res Rev 19:319-325.

Perrier JF, Hounsgaard J (1999) Ca(2+)-activated nonselective cationic current (I(CAN)) in turtle motoneurons. J Neurophysiol 82:730-735.

Rioult-Pedotti MS (1997) Intrinsic NMDA-induced oscillations in motoneurons of an adult vertebrate spinal cord are masked by inhibition. J Neurophysiol 77:717-730.

Rossignol S (1996) Neural control of stereotypic limb movements. In: Handbook of physiology, section 12. Exercise: regulation and integration of multiple systems (Rowell LB, Sheperd JT, eds), pp 173-216. New York: Oxford UP.

Rouse DT, Quan X, McClellan AD (1998) Biophysical properties of descending brain neurons in larval lamprey. Brain Res 779:301-308.

Rovainen CM (1982) Neurophysiology. In: The biology of lampreys, Vol 4A (Hardisty MW, Potter IC, eds), pp 1-136. London: Academic.

Schmidt BJ, Hochman S, MacLean JN (1998) NMDA receptor-mediated oscillatory properties: potential role in rhythm generation in the mammalian spinal cord. Ann N Acad Sci 860:189-202.
Sirota M, Viana Di Prisco G, Dubuc R (1995) Activation of swimming by electrical microstimulation of brainstem locomotor regions in semi-intact lampreys. Can J Physiol Pharmacol 73:A27.

Tsau Y, Wu JY, Hopp HP, Cohen LB, Schiminovich D, Falk CX (1994) Distributed aspects of the response to siphon touch in Aplysia: spread of stimulus information and cross-correlation analysis. J Neurosci 14:4167-4184.

Viala G, Orsal D, Buser P (1978) Cutaneous fiber groups involved in the inhibition of fictive locomotion in the rabbit. Exp Brain Res 33:257-267.

Viana Di Prisco G, Ohta Y, Bongianni F, Grillner S, Dubuc R (1995) Trigeminal inputs to reticulospinal neurones in lampreys are mediated by excitatory and inhibitory amino acids. Brain Res 695:76-80.

Viana Di Prisco G, Pearlstein E, Robitaille R, Dubuc R (1997) Role of sensory-evoked NMDA plateau potentials in the initiation of locomotion. Science 278:1122-1125.

Vinay L, Grillner S (1992) Spino-bulbar neurons convey information to the brainstem about different phases of the locomotor cycle in the lamprey. Brain Res 582:134-138.

Wilson GF, Richardson FC, Fisher TE, Olivera BM, Kaczmarek LK (1996) Identification and characterization of a $\mathrm{Ca}(2+)$-sensitive nonspecific cation channel underlying prolonged repetitive firing in Aplysia neurons. J Neurosci 16:3661-3671.

Zhang B, Wootton JF, Harris-Warrick RM (1995) Ca2+-dependent plateau potentials in a crab stomatogastric ganglion motor neuron. II. Ca2+-activated slow inward current. J Neurophysiol 74:1938-1946.

Zompa IC, Dubuc R (1996) A mesencephalic relay for visual inputs to reticulospinal neurones in lampreys. Brain Res 718:221-227. 